\title{
Isolated metastases of hepatocellular carcinoma in the left atrium, unresponsive to treatment with sorafenib
}

\author{
KAZUYA FUKUOKA ${ }^{1,4}$, ERIKO MASACHIKA ${ }^{2}$, MIKI HONDA ${ }^{2}$, \\ YOSHITANE TSUKAMOTO $^{3}$ and TAKASHI NAKANO ${ }^{2}$ \\ ${ }^{1}$ Department of Medical Oncology, Sakai Hospital, Kinki University Faculty of Medicine, Sakai, Osaka 590-0132; \\ ${ }^{2}$ Division of Respiratory Medicine, Department of Internal Medicine; Departments of ${ }^{3}$ Surgical Pathology \\ and ${ }^{4}$ Genetics, Hyogo College of Medicine, Nishinomiya, Hyogo 663-8501, Japan
}

Received September 12, 2014; Accepted October 22, 2014

DOI: $10.3892 / \operatorname{mco} .2014 .454$

\begin{abstract}
Isolated metastases in the left atrium that are discontinuous with an intrahepatic hepatocellular carcinoma (HCC) are extremely rare. This is the case report of a 46-year-old male patient with pulmonary metastases from HCC, who presented with a tumor in the left lung, extending to the left atrium through the left pulmonary vein. Two weeks after the initiation of treatment with sorafenib, the tumor metastasized to the left parietal cerebral lobe, with an intracranial hemorrhage. Although the patient underwent gamma knife radiosurgery for the metastatic brain tumor, his condition gradually deteriorated and he succumbed to multiple organ failure 4 months later. Given the severe complications that have been reported in patients with this type of metastasis, immediate multidisciplinary treatment, including surgical resection, should be considered.
\end{abstract}

\section{Introduction}

Hepatocellular carcinoma (HCC) is the sixth most prevalent cancer, the third most frequent cause of cancer-related mortality and the leading cause of mortality among patients with cirrhosis (1). Chronic hepatitis B virus (HBV) and hepatitis $\mathrm{C}$ virus (HCV) infection are the most significant causes of HCC. HCCs spread via the hematogenous route, the lymphatic route, or by direct invasion into adjacent organs (2). Metastasis is not rare among patients with $\mathrm{HCC}$ and has increased in prevalence over the last decade (3). HCCs may exhibit multiple intrahepatic occurrences and intrahepatic metastasis, whereas extrahepatic metastasis occurs in $30-50 \%$ of the patients (4). The most common site of extrahepatic metastasis of HCC is

Correspondence to: Dr Kazuya Fukuoka, Department of Medical Oncology, Sakai Hospital, Kinki University Faculty of Medicine, 2-7-1 Harayamadai, Minami-ku, Sakai, Osaka 590-0132, Japan E-mail: yumikofu@m3.kcn.ne.jp

Key words: hepatocellular carcinoma, isolated left atrial metastases, sorafenib, bronchoscopy the lungs, followed by lymph nodes and bones $(3,4)$. Although HCC may metastasize to various extrahepatic organs, metastases with cardiac involvement are rare (5-19). The majority of the cardiac metastases are direct and contiguous extensions of the intrahepatic HCC via the inferior vena cava into the right atrium (16), whereas isolated metastases in the left atrium that are discontinuous with an intrahepatic $\mathrm{HCC}$ are extremely rare $(5,6,9,12)$. This is the case report of a 46 -year-old Japanese man with isolated left atrial metastases from HCC and a review of published reports, treatments and prognoses in similar cases. Written informed consent was obtained from the patient's relatives.

\section{Case report}

A 46-year-old Japanese man was referred to our institution for the treatment of progressive breathlessness and persistent cough over the past 3 months. Two years prior to this visit, the patient had undergone a curative operation for HCC secondary to chronic HBV infection. The patient's blood biochemical values demonstrated elevated levels of serum $\alpha$-fetoprotein (AFP) and protein induced by vitamin $\mathrm{K}$ absence or antagonist-II (PIVKA-II). A contrast-enhanced computed tomography (CT) scan of the patient's chest revealed a tumor extending from the left lower lobe bronchus into the left atrium via the pulmonary vein (Fig. 1). A positron emission tomography scan with ${ }^{18} \mathrm{~F}$-fluorodeoxyglucose $\left({ }^{18} \mathrm{~F}\right.$-FDG) showed increased FDG uptake, with a maximum standardized uptake value of 5.1 in the tumor. There was no abnormal accumulation of FDG in the liver.

A transthoracic echocardiography image revealed a round mass-like lesion in the left atrium (Fig. 2). An endobronchial ultrasound-guided transbronchial needle aspiration (EBUS-TBNA) was performed, targeting the submucosal lesion in the left lower lobe bronchus. The microscopic findings of the specimens obtained by EBUS-TBNA demonstrated polygonal tumor cells with centrally located round nuclei arranged in a predominantly trabecular pattern (Fig. 3A). The tumor was compatible with metastatic HCC, as the cytopathological characteristics of the tumor cells were identical to those of the previously resected HCC, with moderate differentiation (Fig. 3B). Subsequently, oral sorafenib therapy was initiated at 


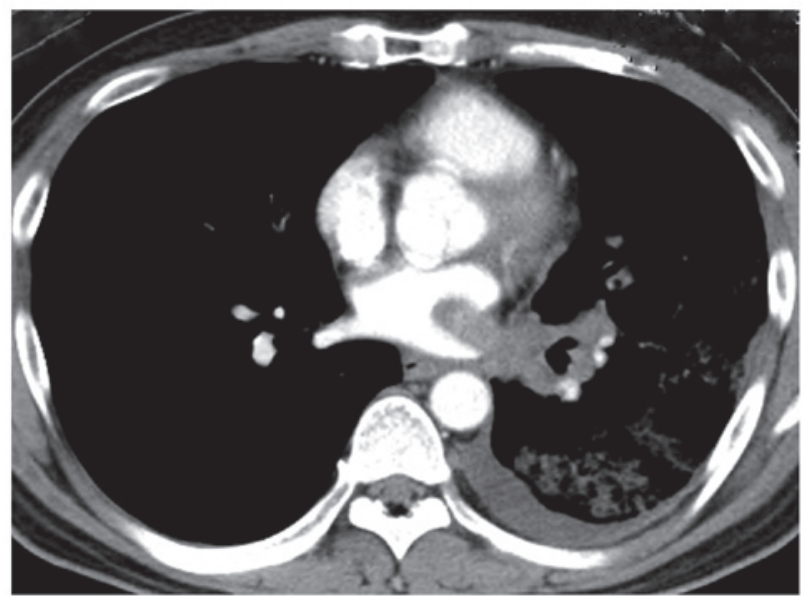

Figure 1. A contrast computed tomography scan of the chest revealed a tumor extending from the left lower lobe bronchus into the left atrium via the pulmonary vein, with nodular opacities in the left lower lobe accompanied by pleural effusion.

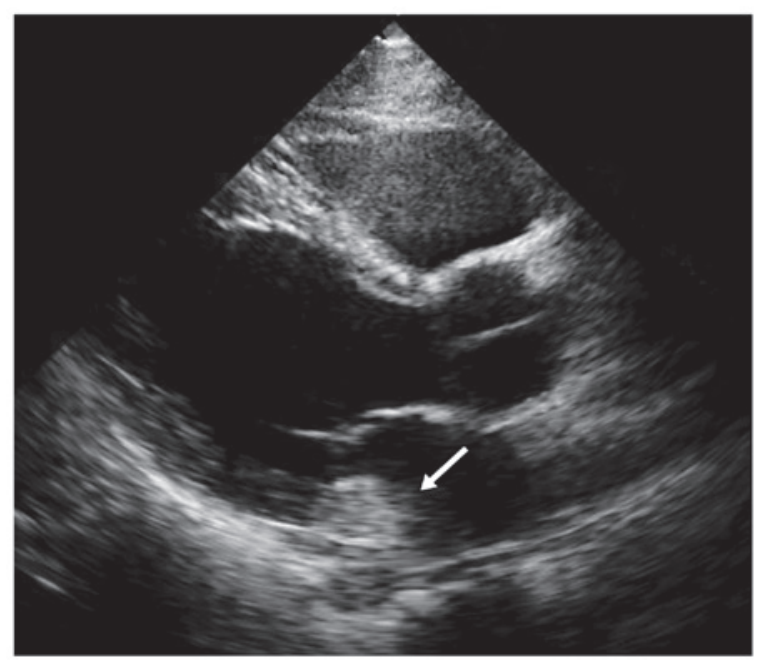

Figure 2. Transthoracic echocardiography image showing a round mass-like lesion in the left atrium (white arrow).

the dose of $400 \mathrm{mg} /$ day in the outpatient setting. Two weeks after the initiation of sorafenib treatment, the patient was admitted to our institution with nausea, vomiting and weakness of the right upper limb. A contrast CT scan of the brain revealed a metastatic brain tumor in the left parietal cerebral lobe with an intracranial hemorrhage. The patient underwent gamma knife radiosurgery for the metastatic brain tumor, following control of the intracranial hemorrhage by conservative treatment and discontinuation of sorafenib. However, the patient's condition gradually deteriorated and he succumbed to multiple organ failure 4 months later.

\section{Discussion}

To the best of our knowledge, this is the first reported case of isolated metastases of HCC to the left atrium treated with sorafenib. Metastases of HCC tend to spread through the intrahepatic vessels, the lymphatic system, or by direct invasion. HCC has a marked propensity for vascular invasion

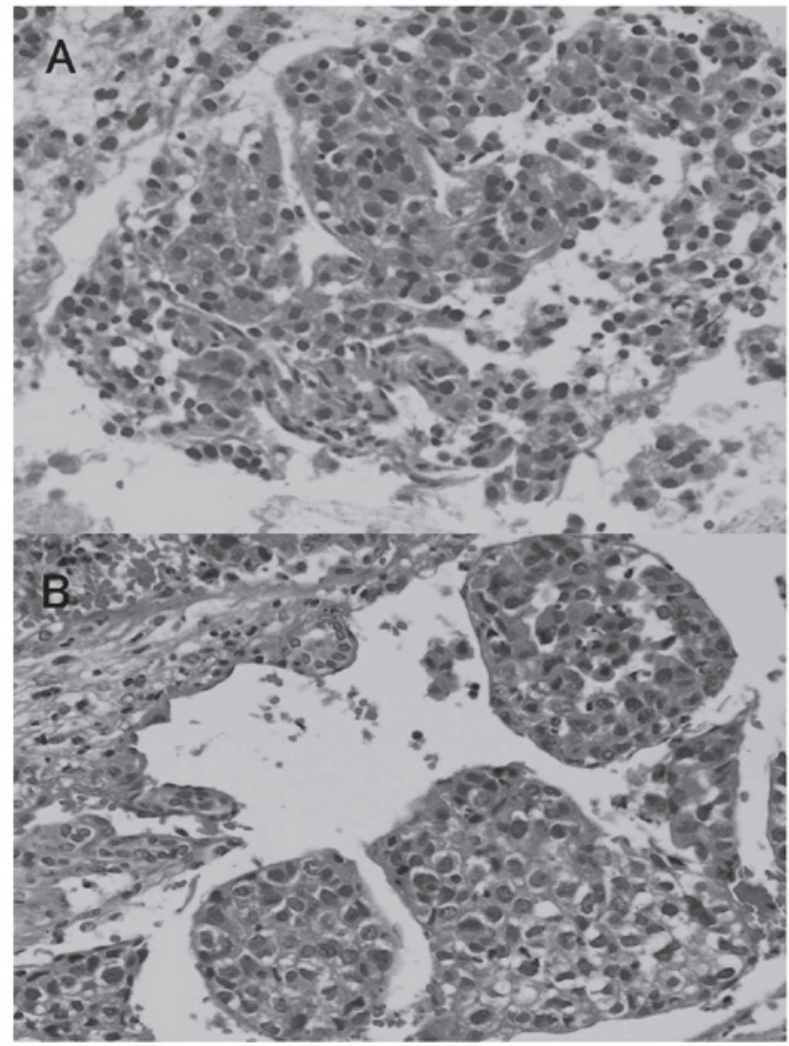

Figure 3. Microscopic findings of biopsy and surgical resection specimens. (A) Microscopic findings of specimens obtained by endobronchial ultrasound-guided transbronchial needle aspiration, showing polygonal tumor cells with centrally located round nuclei arranged in a predominantly trabecular pattern (hematoxylin and eosin staining; original magnification, x100). (B) Microscopic findings of resected specimens, exhibiting moderately differentiated hepatocellular carcinoma of the trabecular type (hematoxylin and eosin staining; original magnification, $\mathrm{x} 100$ ).

and extension. Hematogenous spread may result from the involvement of the portal vein, hepatic veins, or inferior vena cava (18). Although HCC may metastasize to various extrahepatic organs, metastases with cardiac involvement are rare (12). The majority of cardiac metastases are direct and contiguous extensions of the intrahepatic HCC via the inferior vena cava into the right atrium (16).

In an antemortem series, the incidence of metastatic HCC to the right heart cavity with extension via the inferior vena cava was reported to be $<6 \%$ (5). Isolated cardiac metastases that are discontinuous with an intrahepatic HCC are extremely rare; to the best of our knowledge, a total of 18 cases with isolated cardiac metastases from $\mathrm{HCC}$ have been reported to date $(14,16)$. In those cases, the majority of metastases were located in the right side of the heart, while metastases in the left atrium were observed in only 2 patients. The involvement of the left atrium is possibly associated with tumor growth from the pulmonary veins following metastasis to the lung, direct invasion of the atrial septum, or tumor implantation via a subclinical right-to-left shunt through a patent foramen ovale.

In the present case, the lung metastases of HCC may be considered to be the origin of the patient's metastatic tumor in the left atrium. It is hypothesized that the tumor cells hematogenously spread from the tumor located in the lung via the pulmonary veins directly into the left atrium, as the 
echocardiography and CT scan did not reveal a patent foramen ovale or other cardiac defect.

The risk of cardiopulmonary collapse is high in patients with cardiac involvement. Possible cardiopulmonary complications include heart failure, tricuspid stenosis or insufficiency, ventricular outflow tract obstruction, sudden cardiac death, secondary Budd-Chiari syndrome, pulmonary embolism and pulmonary metastasis (19). Acute severe complications, including cerebral infarction, peripheral arterial occlusion and syncopal attack have also been reported, as left atrial masses may give rise to widespread emboli (20). In the present case, the tumor cells in the left atrium may have spread hematogenously to the brain via the systemic circulation, resulting in tumor emboli and a cerebral hemorrhage. Brain metastases from $\mathrm{HCC}$ are extremely rare, with a reported frequency ranging from 0.2 to $2.2 \%$ at autopsy, and they are frequently associated with hemorrhage (21). In general, the hypervascularity of HCCs and the underlying coagulopathy due to liver cirrhosis may account for cerebral hemorrhage (21).

Multidisciplinary treatments to control the growth of HCC offer patients with cardiac involvement a potentially curative option. Surgical and non-surgical approaches, including transcatheter arterial embolization and transcatheter arterial infusion chemotherapy, have been undertaken to treat patients with isolated cardiac metastases from HCC $(14,16)$. However, such therapeutic modalities may not be feasible, particularly if the patient has a poor general status, metastatic disease, or underlying hepatic dysfunction (17). Until recently, no effective treatment was available for HCC patients diagnosed at an advanced stage, or who progressed to an advanced stage following failure of other treatments (1).

Several molecular-targeted agents are currently under development, but the only one with proven survival benefit is sorafenib (1). This agent, which may be administered orally, is a multikinase inhibitor that blocks Raf signaling and vascular endothelial growth factor (VEGF), platelet-derived growth factor (PDGF) and c-Kit (1). Recent studies have established sorafenib as the standard of care for advanced HCC (1). Since an aggressive surgical resection of the tumor was not feasible for our patient due to the advanced stage of the HCC, treatment with sorafenib was initiated to prolong his survival. However, sorafenib was ineffective and provided no survival benefit in this case. It is possible that the growth mechanisms of this tumor do not depend on Raf, VEGF, PDGF or c-Kit signaling, but rather on an alternative signal transduction pathway, resulting in failure of sorafenib treatment.

The prognosis of HCC with cardiac involvement is poor $(14,16,17)$. The reported median survival time from the time of diagnosis of cardiac metastasis was 102 days (17). The most common cause of death is usually associated with the HCC per se or the underlying liver disease; only a few patients reportedly succumb to cardiac metastases (17).

In conclusion, we treated a patient with HCC and isolated metastases in the left atrium. Isolated metastases in the left atrium that are discontinuous with an intrahepatic HCC are extremely rare. Given the severe complications that have been reported in patients with this type of metastasis, immediate multidisciplinary treatment, including surgical resection, should be considered.

\section{References}

1. Forner A, Llovet JM and Bruix J: Hepatocellular carcinoma. Lancet 379: 1245-1255, 2012.

2. Becker AK, Tso DK, Harris AC, Malfair D and Chang SD: Extrahepatic metastases of hepatocellular carcinoma: a spectrum of imaging findings. Can Assoc Radiol J 65: 60-66, 2014.

3. Abbas A, Medvedev S, Shores N, et al: Epidemiology of metastatic hepatocellular carcinoma, A Nationwide Perspective. Dig Dis Sci 2014

4. Terada T and Maruo H: Unusual extrahepatic metastatic sites from hepatocellular carcinoma. Int J Clin Exp Pathol 6: 816-820, 2013.

5. Lei MH, Ko YL, Kuan P, Lien WP and Chen DS: Metastasis of hepatocellular carcinoma to the heart: unusual patterns in three cases with antemortem diagnosis. J Formos Med Assoc 91: 457-461, 1992.

6. Barasch E, Frazier OH, Silberman H, Shannon RL and Wilansky S: Left atrial metastasis from hepatocellular carcinoma: a case report. J Am Soc Echocardiogr 7: 547-549, 1994.

7. Masci G, Magagnoli M, Grimaldi A, et al: Metastasis of hepatocellular carcinoma to the heart: a case report and review of the literature. Tumori 90: 345-347, 2004.

8. Longo R, Mocini D, Santini M, et al: Unusual sites of metastatic malignancy: case 1 . Cardiac metastasis in hepatocellular carcinoma. J Clin Oncol 22: 5012-5014, 2004.

9. Nam SW, Baek JT, Kang SB, et al: A case of the hepatocellular carcinoma during the pregnancy and metastasis to the left atrium. Korean J Hepatol 11: 381-385, 2005.

10. Agelopoulou P, Kapatais A, Varounis C, et al: Hepatocellular carcinoma with invasion into the right atrium. Report of two cases and review of the literature. Hepatogastroenterology 54: 2106-2108, 2007

11. Leo F, Rapisarda F, Stefano PL and Batignani G: Cavo-atrial thrombectomy combined with left hemi-hepatectomy for vascular invasion from hepatocellular carcinoma on diseased liver under hypothermic cardio-circulatory arrest. Interact Cardiovasc Thorac Surg 10: 473-475, 2010.

12. Jeong DS, Kim JS, Kim KH and Ahn H: Left atrial metastasis from hepatocellular carcinoma with liver cirrhosis. Interact Cardiovasc Thorac Surg 11: 703-705, 2010.

13. Inoue $Y$, Hayashi M, Katsumata T, Shibayama $Y$ and Tanigawa N: Hepatocellular carcinoma with right atrial tumor thrombus: report of a case. Surg Today 41: 1122-1129, 2011.

14. Tastekin E, Usta U, Ege T, Kazindir G and Kutlu AK: Cardiac metastasis of hepatocellular carcinoma in a young non-cirrhotic patient, to the left ventricle. Ann Hepatol 11: 392-394, 2012.

15. Li SS, Jian LL, Gang PB, Ando KM and Ming LD: Removal of hepatocellular carcinoma extending into the right atrium with extracorporeal circulation. Hepatogastroenterology 59: 1591-1593, 2012.

16. Kawakami M, Koda M, Mandai M, et al: Isolated metastases of hepatocellular carcinoma in the right atrium: case report and review of the literature. Oncol Lett 5: 1505-1508, 2013.

17. Albackr HB: A large right atrial mass in a patient with hepatocellular carcinoma: case report and literature review. J Saudi Heart Assoc 26: 174-178, 2014.

18. Liu YC, Ho YL, Huang GT, Chen DS, Sheu JC and Chen CH: Clinical manifestations and survival of patients with hepatocellular carcinoma and cardiac metastasis. J Gastroenterol Hepatol 25: 150-155, 2010.

19. Sung AD, Cheng S, Moslehi J, Scully EP, Prior JM and Loscalzo J: Hepatocellular carcinoma with intracavitary cardiac involvement: a case report and review of the literature. Am J Cardiol 102: 643-645, 2008.

20. Funakoshi Y, Mukohara T, Kataoka T, et al: Left atrial extension of metastatic lung tumor via pulmonary vein: report on the first case of Ewing sarcoma. Rare Tumors 2: e53, 2010.

21. Jiang XB, Ke C, Zhang GH, et al: Brain metastases from hepatocellular carcinoma: clinical features and prognostic factors. BMC Cancer 12: 49, 2012. 